\title{
Linx
}

Revue des linguistes de l'université Paris X Nanterre

9 | 1997

Émile Benveniste. Vingt ans après

\section{Benveniste et la catégorie du romanesque : Greimas, Weinrich, Larbaud, Dujardin}

\section{Francis Gandon}

\section{(2) OpenEdition}

\section{Journals}

Édition électronique

URL : http://journals.openedition.org/linx/1029

DOI : 10.4000/linx.1029

ISSN : 2118-9692

Éditeur

Presses universitaires de Paris Nanterre

\section{Édition imprimée}

Date de publication : 1 avril 1997

Pagination : 169-178

ISSN : 0246-8743

\section{Référence électronique}

Francis Gandon, «Benveniste et la catégorie du romanesque : Greimas, Weinrich, Larbaud, Dujardin », Linx [En ligne], 9| 1997, mis en ligne le 05 juillet 2012, consulté le 30 avril 2019. URL : http:// journals.openedition.org/linx/1029; DOI : 10.4000/linx.1029 


\title{
Benveniste et la catégorie du romanesque : GREIMAS, WEINRICH, LARBAUD, DUJARDIN
}

\author{
Francis Gandon
}

$\mathrm{N}$

ous considérerons, dans cette communication, la confrontation entre Benveniste et Weinrich comme présentant peu d'intérêt en soi. Elle met, en revanche, sur la voie d'une recherche qui outrepasse les bornes du linguistique, pour s'adresser au domaine littéraire.

Nul n'ignore que, chez Benveniste, avec l'histoire et le discours, une rigoureuse dichotomie structure l'énonciation à la fois au plan des tiroirs verbaux et à celui des pronoms. Il n'a cependant guère été vu que Benveniste se débarrasse de ce qui, de quelque façon, manque à l'une et l'autre classes, dans une catégorie annexe, dont, à vrai dire, il ne fait qu'esquisser les traits: le "romanesque".

Nous aimerions montrer, ici, en quoi le retour de ce que la réduction binaire a rejeté s'effectue à travers précisément le texte littéraire, et, notamment l'un de ses traits: le discours indirect libre. Benveniste nous conduira - dans une sorte de chronologie inversée - à Weinrich et Greimas, d'une part, de l'autre à Émile Dujardin (1868-1946) et Valéry Larbaud (1881-1957), avec, en toile de fond, la figure emblématique de William Joyce (1882-1941).

La dichotomie benvenistienne, dont on connaît la fortune, a été, on le sait moins, revue et corrigée par ce spécialiste de la "linguistique textuelle" qu'est Harald Weinrich. Que faut-il comprendre sous l'expression? Si le projet recommande - suivant en cela les déclarations déjà anciennes de L. Hjelmslev (1943) - de prendre pour unité le seul texte, la pratique descriptive du livremanifeste Le Temps (1963) se limite de manière quasi exclusive aux formes verbales. Que la discipline ainsi conçue ait accédé à la reconnaissance académique [H. Weinrich a occupé la chaire de "linguistique textuelle" au Collège de France en 1991], est, d'autre part, symptomatique de l'intérêt porté au système verbal comme "module" autonome, si l'on nous passe cet anachronisme. 


\section{Francis Gandon}

La confrontation Benveniste/Weinrich conduit d'abord à analyser la différence entre les descriptions. Cette différence nous mettra sur la voie d'une problématique décentrée : celle du "romanesque", qui perturbe, en retour, de manière irrémédiable, la pure architecture benvenistienne.

\section{Querelle de personne}

On sait que Benveniste oppose de façon cardinale deux modes d'énonciation: l'histoire et le discours (1959); le premier, qui exclut toute marque d'un sujet narrateur au profit d'événements qui "semblent se raconter d'eux-mêmes" (1959: 241), recourt à des tiroirs verbaux privilégiés : 1' "aoriste" (passé simple) et l'imparfait. Sa personne de prédilection est le il. Le second se définit inversement comme "toute énonciation supposant un locuteur et un auditeur et, chez le premier, l'intention d'influencer l'autre en quelque manière" (1950 : 241-242) ; il renvoie à une énonciation qu'instancient des "déictiques" (je, $i c i$, maintenant, hier $)^{1}$... qui forment un sous-ensemble s'opposant à d'autres indicateurs (il, là, alors, la veille...). Il recourt à l'ensemble des tiroirs verbaux à l'exception du passé simple.

H. Weinrich (1963) dispose, quant à lui, une dichotomie analogue entre temps narratifs et commentatifs. Chacun des groupes se caractérise par une récurrence "obstinée" de morphèmes temporels. Ainsi, en français, les temps commentatifs comportent-ils le passé composé, le présent, le futur et le futur antérieur. Tous les autres tiroirs relèvent des temps narratifs. Chacun témoigne d'une "attitude de locution": détente (c'est le cas pour un texte à dominante narrative) ou tension (quand, inversement, le commentaire l'emporte). La chose est assez logique puisque ce qui relève du commentaire est censé poursuivre ses effets jusqu'au moment de l'énonciation.

Se disant "redevable de mille suggestions" à un Benveniste qualifié d' "aussi fin observateur qu'analyste pénétrant" (1963: 61), Weinrich met cependant un bémol à cette esquisse de dithyrambe : "Dans ma conception, un seul et même temps ne pourra jamais appartenir à la fois au groupe du commentaire (que l'on peut sans doute rapprocher du "discours" de Benveniste) et au groupe du récit (peut-être comparable à ce qu'il appelle histoire)" (1963:62).

Si cette bipartition rigoureuse peut, après tout, se justifier, c'est cependant avec étonnement qu'on lit, un peu plus bas, ce qui suit: "Autre divergence : je n'admettrai pas qu'un temps puisse passer d'un groupe à l'autre

\footnotetext{
${ }^{1}$ Qui équivalent aux "embrayeurs" (traduction, par N. Ruwet de shifter, dont la paternité revient à Jespersen). À noter un déplacement terminologique puisque, dans le cadre jakobsonien, les "déictiques" constituent un sous-ensemble caractérisé par la nécessité d'une information supplémentaire: ils correspondent à ce que Benveniste désigne comme marques de l'ostension (1970 : 82). Signalons enfin qu' "embrayeur" est parfois utilisé pour désigner tout repère: par exemple le lendemain aussi bien que demain.
} 
selon la personne avec laquelle il se combine" (ibid). Ici le lecteur se récrie : à relire l'article fondamental: "Les relations de temps dans le verbe français" (1959), cette soi-disant migration en fonction de la personne procède d'une lecture fautive. Benveniste n'ignore évidemment pas l'autobiographie, et précise en note que c'est le romancier qui "emploie encore sans effort l'aoriste aux premières personnes du singulier et du pluriel" (1959: 244, n.3) - en soulignant une différence de statut entre lui et l'historien, différence qu'une note précédente $(1959: 242, n .2)$ envisageait déjà : Benveniste y explicitait très lucidement le choix de la terminologie de "récit historique", en lieu et place de "temps narratif". C'est qu'en réalité la bipartition benvenistienne ne sature pas l'ensemble des combinaisons formes verbales/pronoms. Pour que la théorie rende compte de l'ensemble du système, une troisième catégorie est nécessaire (et postulée) : la catégorie "romanesque". (À voir la fortune des modèles binaires ultérieurs, se réclamant peu ou prou de Benveniste, on mesure à quel point un article, de prime abord aussi transparent, a été mal compris - et ceci en proportion même des difficultés qu'il comporte; d'une façon générale, Benveniste récuse tout simplisme pédagogique, ce qui fait de ses paires oppositionnelles des dualités décevantes ou fausses: ainsi l'autre couple canonique : énoncé/énonciation, n'en est pas vraiment un).

La lecture fautive de Weinrich n'en est pas moins hautement significative, en ce qu'elle concerne la catégorie de la personne, oblitérée chez lui, tandis qu'elle fait, chez Benveniste, l'objet d'une appropriation théorique brillante et définitive : l'opposition de la "non-personne" (le il) à l'axe je-tu. Brillante, mais probablement mal assumée: en ceci Weinrich, à son corps défendant, retourne le couteau dans la plaie théorique, comme nous aimerions à présent le montrer.

Arrêtons-nous un instant sur la donnée suivante : qu'y-a-t-il de troublant, dans la collusion aoriste/première personne, au point que Benveniste crée, tout exprès pour elle, une catégorie annexe et quelque peu clandestine?

Ce qu'en réalité Benveniste n'envisage pas, c'est qu'un récit de type autobiographique mette en place un dispositif tensionnel entre deux "je" : un "je" de l'énoncé - projeté dans l'objectivité du récit à laquelle il se voue par une sorte d'amputation schizoïde - et un "je" de l'énonciation" ${ }^{2}$, dont le propre est d'instancier le narré, de le repérer, le renvoyer à une instance, donc de suturer la coupure (ce qui "expliquerait" l'appariement symétriquement électif du parfait et de la première personne ${ }^{3}$ ). On sait qu'en revanche un des avatars les

2 Une raison possible de cette occultation : contrairement à d'autres (duel, triel, nous inclusif vs exclusif .. ), le distinguo ne semble pas être pris en charge par la grammaire des langues naturelles. Cf. Normand souligne, ici même, l'absence d'une théorie du sujet de l'énonciation chez Benveniste.

${ }^{3}$ Un contre-exemple frappant est fourni par certaines langues naturelles, dont les tiroirs, relevant des deux "univers énonciatifs" discriminés tant par Weinrich que Benveniste de façon catégorique, peuvent s'accoler dans des groupes fortement cohésifs, par exemple la phrase grecque ha egrapsa guegrapha, littéralement : ce que j'écrivis (action passée), je l'ai écrit (résultat). 


\section{Francis Gandon}

plus puissants de la linguistique structurale, la sémiotique greimassienne, envisage explicitement une telle scission sous le terme de débrayage : "opération par laquelle l'instance de l'énonciation disjoint et projette hors d'elle, hors de l'acte de langage et en vue de la manifestation, certains termes" (A.J. Greimas et J. Courtès, 1979 : 79).

Dans cette optique les embrayeurs ne sont plus seulement les outils d'une métalangue, mais les actants d'une opération ("embrayage") symétrique, on ne peut plus logiquement, à l'opération inverse ("débrayage") non envisagée, semble-t-il, par la linguistique.

Toutefois une telle transparence se trouble à l'usage. Greimas, en effet, avait antérieurement pris soin de désigner les marques de l'énonciation (déictiques, pronoms ... ) comme des simulacres. Mais, surtout, il avait introduit un relativisme radical, qui privait de positivité la dimension pronominale. En effet le tandem je-tu n'introduisait qu'une "illusion de réalité", et, dans tel texte d'H. Michaux "Il n'[était] pas possible d'affirmer que Michaux [fût] moins présent dans le "il" de la première partie que dans le "je" de la seconde" (1972: 20). Assez curieusement, la définition de 1979 sera en retrait sur cette position. Si les pronoms continuent à être considérés comme des "simulacres" (mais cela est-il si significatif : vis à vis de l'ineffable, tout est "simulacral" : "La nuit, toutes les vaches sont noires", pour reprendre l'aphorisme hégélien visant Schelling), Greimas respecte, en gros, le dichotomie benvenistienne fondatrice entre l'axe je-tu et la "non-personne" (1979: 79-82).

Retrait, en effet, pas forcément visible, puisque je simulacre de JE ("véritable énonciateur"), tu de TU - mais je/tu clivés de $i l$ - est une proposition très différente de celle qui affirme que je, aussi bien que il, est le simulacre de JE.

Il s'agit là, en termes weinrichiens, d'une transition de deuxième degré : concernant la perspective de locution (anticipation/rétrospection) et l'attitude de locution (récit/commentaire), soit une "métaphore temporelle". Evidemment prévue comme procédure de médiation entre énoncés, la métaphore ne fonctionne pas, en principe, comme mécanisme de constitution de l'énoncé de base : ainsi l'unité minimale de discours qu'est la phrase.

Certes, Benveniste précise que la division en question concerne les tiroirs verbaux $d u$ français ; on pourrait, par ailleurs, nous reprocher de projeter une problématique "moderne" sur un contexte qui n'en a que faire, et de négliger la grammaire historique (un helléniste pourrait distinguer des périodes de plus ou moins grande diffusion de tel ou tel tiroir). Il n'en reste pas moins que les valeurs du parfait et de l'aoriste grecs correspondent de manière étonnante à celles des passés composé et simple du français. Il n'est d'ailleurs pas indifférent que Benveniste ait utilisé une terminologie grecque, au fond saugrenue. Ainsi, le paradigme histoire/récit a-t-il bien vocation à constituer un fait de grammaire générale (cf les nombreux recours, chez Weinrich, à des langues européennes modernes), et c'est peut-être la combinaison tiroirs/pronoms, qui resterait à affiner mais pas dans le sens indiqué par la linguistique textuelle. Signalons enfin qu'en français, la transition passé simple/passé composé, outre les cas orthodoxes, est extrêmement banale, mais que, d'une part, elle n'est pas en règle générale constitutive d'énoncés; qu'elle opère, d'autre part, de façon peu visible, "clandestine" (du moins en français moderne. Un texte comme Le Roman de Renart constitue, à l'inverse, une mine inépuisable de transitions "hétérodoxes" explicites).

Rappelons enfin que la phrase prononcée par Pilate : ho guegrapha guegrapha est considérée comme un calque du latin quod scripsi scipsi (avec le ho (=quod) au lieu de $h a$ ). Le fait que le rédacteur ait pris soin de noter une telle "incorrection" va dans le sens d'un témoignage direct, à l'encontre de la thèse (à vrai dire de moins en moins soutenue) d'une rédaction tardive des Évangiles. 
Une telle oscillation, qui implique manifestement quelque chose de l'ordre de la passion, incite à considérer comme également passionnée la si rigoureuse architecture, dont la linguistique reste redevable à l'enfant d'Alep.

Résumons.

- L'auteur des Noms d'agent... maintient rigoureusement séparés ses deux "univers énonciatifs" au plan de ce qu'on pourrait appeler - anachronisme voulu - les "modules" pronominal et déictique. Au positivisme de la dichotomie répond la nature à la fois pleine et littérale des pronoms. Ce qui ne correspond pas au rangement binaire (par exemple la co-présence de je et de l'aoriste) est évacué dans une troisième catégorie - impure - non linguistique, qu'on peut grossièrement qualifier de "romanesque".

- L'auteur de Maupassant... envisage une béance entre l'énonciation "authentique" et son simulacre textuel. Mais l'énonciation "authentique" étant de l'ordre de l'ineffable, du mystique, ou encore de la "théologie négative" (G. Bataille), - cette béance est elle-même, à peu près, simulacrale.

- En revanche la non-différenciation (en 1972) du je et du il grammaticaux introduisait une rupture dans l'ordre énonciatif, peut-être trop radicale pour que Greimas ne la mentionnât pas qu'en passant, pour n'y plus faire allusion par la suite.

- Rappelons enfin, pour mémoire et par souci d'exhaustivité, que la dualité énonciative ne se supporte, chez Weinrich, que d'une bipartition verbale. Un tel "positivisme du tiroir" nous paraît largement insatisfaisant ${ }^{4}$.

Benveniste dans tout cela? Notre interprétation est la suivante: la rigoureuse schize du module pronominal-déictique est une autre façon d'affirmer le caractère positif et littéral $d u$ pronom - donc de nier la possibilité d'un méta-pronom - donc de nier la possibilité d'un inconscient - inconscient dont les opérations - condensation et déplacement pour s'en tenir à des valeurs sûres - brouillent de façon en quelque sorte canonique l'Ordre pronominal.

\section{Les lauriers sont coupés}

Or, à s'en tenir à un domaine linguistique - plus précisément aux franges du linguistique et du littéraire - il est un mode de discours pour contester l'arrogance du clivage et fournir l'un des rares cas d'un dialogue en quelque sorte expérimental entre énonciation et énoncé, où quelque chose s'exprime, de façon impersonnelle et irresponsable : le discours indirect libre.

\footnotetext{
${ }^{4}$ D'une façon générale, la description du système verbal des langues naturelles peut difficilement faire abstraction des pronoms. Beaucoup d'idiomes présentent une étroite imbrication entre les deux sous-systèmes. Ainsi, dans de nombreuses langues africaines, les pronoms sont-ils des éléments de formation des divers "temps" du verbe. C'est par exemple le cas du baatonum (langue voltaïque, voir R.P. A. Prost, 1979), du hausa (langue tchadique, voir J.G. Malka, 1990). En wolof (groupe ouest-atlantique) on ne peut parler de paradigmes distincts pour le temps et la personne, les marques se caractérisant par un syncrétisme généralisé (voir A. Dialo, 1983).
} 


\section{Francis Gandon}

Avec ce "style", comme on disait, le il vaut pour un je, et les cartes sont brouillées au point qu'on peut ne plus savoir qui parle : Flaubert est, on le sait, un spécialiste du genre.

Le discours indirect libre neutralise à la fois la distinction entre personnes et le contraste entre embrayeurs et marques de l'histoire. Ainsi, dans l'exemple :

1) Elle se rasséréna. Bien entendu, demain elle n'irait pas le voir.

l'adverbe de temps pourrait-il être remplacé par le lendemain. On considérera, au passage, comme une curiosité le fait que les marques de l'histoire soient plutôt utilisées dans le sens du passé que dans celui du futur. Que l'on compare :

2) Elle se rasséréna. La veille, n'avait-elle pas revu Firmin?

\section{[ ?] Hier}

(où l'on est tenté de faire précéder hier d'un point d'interrogation)

et :

3) Elle se rasséréna. [ ?] Le lendemain, ne verrait-elle pas Firmin ?

Demain

(où, cette fois, c'est la première formule qui paraît nettement insolite).

On peut donc considérer la neutralisation entre classes d'indicateurs comme non pas automatique mais orientée : prenant en charge la direction du temps vers le hic et nunc de l'énonciation (favorisant les embrayeurs dans cette direction, les défavorisant dans le sens contraire). Elle est, d'autre part, attestée en littérature de façon récurrente :

4) Notre héros eut le malheur de s'arrêter auprès de cette petite chaise de paille, qui jadis avait été le témoin de triomphes si brillants. Aujourd'hui personne ne lui adressa la parole ${ }^{5}$.

Elle peut même constituer le principe d'une technique narrative conduite systématiquement, comme c'est le cas pour un curieux récit de Valéry Larbaud,

\footnotetext{
${ }^{5}$ Stendhal, Le Rouge et le Noir, cité par J. Moeschler, 1993. L'interprétation de tels faits par la pluralisation des fictions (fiction principale, fiction secondaire selon Vuillaume, 1990) ne nous parait guère satisfaisante. Dans une perspective générativiste, ce type de discord pourrait être traité en termes de noeud E(expression) (A. Banfield, 1973) ou de noeud Qualité (J. Cl. Milner, 1978 ; N. Ruwet, 1982).
} 
Mon plus secret conseill'. Le thème de l'histoire est la "fuite" d'un homme excédé par une maîtresse qu'il a fini par abandonner à Naples. Erratique et ferroviaire, le voyage s'agrémente de nombreuses rétrospections, de réflexions (sur les femmes en général, les maîtresses en particulier), de plusieurs décisions prises pour l'avenir, et de certaines palinodies : ne ferai-je pas mieux de revenir ? La pensée du narrateur - mais lequel ? - semble rythmée par le mouvement du train et les arrêts-départs constants. La narration passe alternativement du je au $i l$, sans que cette différence de débrayage corresponde à une modification détectable, au plan des contenus, et notamment à celui de la compétence cognitive: ce savoir - délégué au lecteur - ne s'élargit nullement avec la troisième personne, symétriquement, les passages en je n'ont rien de particulièrement introspectif. À y regarder d'un peu près, en ce qui concerne la dominance de telle ou telle personne, les chapitres se distribuent de la façon suivante (il n'est évidemment pas tenu compte des passages explicitement donnés comme relevant du discours direct, du monologue intérieur ou de la citation) 1 : il-je. 2 : il. 3 : je. 4 : il. 5 : il-je. 6 : il. $7:$ je-il. $8:$ il-je. $9:$ il. $10:$ il. 11 : il-je. 12 : je. 13 : il. 14 :je. 15 : je. 16 : il-je. 17 : je. $18:$ tu. 19 : tu-,je il. 20 : je. 21 : je (ce dernier chapitre, en vers libres, serait assez proche - au moins formellement de l'expression lyrique).

Comme on peut le voir, certains chapitres privilégient une personne de manière exclusive. D'autre part, les transitions sont assez aisément détectables : ainsi le passage du je au il (7 et 19), ou du il au je $(1,8,11,16)$. La transition d'un chapitre à l'autre est souvent assez nette ( 2 à 3,12 à 13$)$. Toutefois les raisons ?

Il arrive que ce soit l'arrêt à une nouvelle gare qui coïncide avec le changement de personne: le rythme (pseudo) énonciatif se moule sur le rythme de la machine. Ainsi au chapitre 17 (je souligne) :

Et cela m'a rapproché de la pensée d'Irène [... ]

Baragiano.

Mais avec Irène, il se passait de l'intermédiaire des sens [... ] (p. 704).

Picemo.

Ou au chapitre 19 :

Tito.

Et si tu la dédaignais, ne le regretterais-tu pas plus tard? [Le personnage s'adresse ensuite à lui-même] Et puis, assez! je l'aime! je l'aime, et je veux plaider ma cause, et je veux me lier à cette délicieuse colonne (la femme vue de côté, comme dans la danse: le beau flanc, le pas suspendu, un bras levé). Ralentissement-aiguillage -àcoups - plusieurs voies - une grande gare défile - buffet.

Potenza.

Ah, c'est ça, Potenza? Il a le temps d'aller au buffet manger un sandwich et boire un café chaud. Pas envie de ce qu'il y a dans ce panier [... ] (pp. 708-709).

6 1922-1923, Nouvelle revue française, septembre-octobre 1923. Cité «d'après l'édition de la Pléïade (G. Jean-Aubry, R. Mallet, 1984 (premier dépôt légal : 1957), avec une préface de M. Arland). 
Francis Gandon

La simplicité du procédé n'est qu'apparente : ainsi, dans le chapitre 17, un débrayage interne a-t-il déjà provoqué une transition je-il. Larbaud échappe au reproche de maniérisme en ce que, hormis quelques exemples détectables, un changement de gare ${ }^{7}$ n'a pas de conséquence "énonciative" systématique (heureusement...). Souvent, rien ne permet d'expliquer la mutation pronominale. Par ailleurs, l'insertion de passages en je à l'intérieur de séquences en $i l$, et dans la mesure où ces dernières semblent relever du discours indirect libre, font du je non pas l'expression d'une spontanéité (d'un "discours direct"), mais, de façon fort retorse, un il déguisé : il s'agit, en ce sens, d'une fausse "transition" (au sens weinrichien). Ainsi : " Vraiment, il ne doutait de rien en ce temps-là. Eh bien, ne suis-je pas le même ? Ne puis-je me délivrer quand je veux du réseau que mes actions ont tissé autour de moi ? Certainement. Examine avec impartialité les pièces du procès. La première de toutes...j'ai honte. Non : pas de respect humain, la vérité." (p. 668). On aura remarqué la présence d'un $t u$ renforçant la fiction d'un monologue intérieur, monologue donnant lieu à un ruissellement qui s'achève en la cataracte rythmique du chapitre final, déjà mentionné. La seconde personne domine un chapitre (18) et occupe une place notable dans un autre (19). L'assez effrayante complexité des procédures d'embrayage/débrayage n'est pas sans évoquer ce qui se passe en langue naturelle. Le sentiment qui domine est cependant la perplexité : même si le il est assignable à un énonciateur abstrait, n'en est pas pour autant élargi le faire interprétatif propre au je (ou au $t u$ ou encore au $i l$ censé relever du discours indirect libre). De là à inférer que la personne importe peu, ou pas du tout...

Il est clair cependant qu'il faut exclure une gratuité totale : il reste alors à tenter d'expliquer le pourquoi d'un tel gongorisme de l'énonciation énoncée. Il se trouve une esquisse d'explication au plan intertextuel. La nouvelle porte, en effet, la dédicace suivante :

\section{$A$}

Édouard Dujardin, auteur de

Les Lauriers sont coupés (1887) a quo...

\footnotetext{
7 À signaler aussi que le rythme du voyage peut introduire une rupture à l'intérieur de syntagmes fortement cohésifs :

"nous sommes aussi repu

"Oh, l'amie, la voix de$$
\text { d'amour" (p. 692) }
$$

Persano.

Contursi

l'amie (p. 693)

Technique expressionniste qui n'est pas sans évoquer la Prose du Transsibérien de B. Cendrars (1913).
} 
Or cette dernière expression, qui laisse entendre une origine réelle, renvoie, en fait, à une naissance mythique : elle laisse entendre qu'à partir de la date indiquée, quelque chose devient possible, quelque chose qui ressort de la stupéfaction de la rencontre ${ }^{8}$. Échange de bons procédés, potlatch de l'imaginaire: Larbaud préfacera l'édition définitive (1924) de Les lauriers sont coupés... Or les notes de G. Jean-Aubry et R. Mallet (édition Pléiade) nous apprennent qu'en 1920 Larbaud rencontra, pour la première fois, James Joyce, qui lui parla de Les lauriers sont coupés d'Édouard Dujardin, comme l'une des sources d'Ullysses. En 1923, Larbaud écrit à Dujardin ces lignes étonnantes : "[Les lauriers sont coupés] est non seulement le premier livre écrit en monologue intérieur - ce qui constitue une trouvaille littéraire d'une importance incalculable - mais aussi un parfait chef d'oeuvre, à mettre auprès des plus grands romans de la littérature française! Je suis stupéfait de penser que cela date de 1887 et qu'il a fallu attendre jusqu'à Ulysses pour que quelqu'un reprît la forme du monologue intérieur. Actuellement toute une littérature des États-Unis en est pleine ; votre influence, à travers James Joyce, se fait sentir dans toute l'Amérique. Et moimême, j'ai écrit ce que j'ai cru être, - jusqu'à ma première lecture de Les lauriers sont coupés, - les deux premiers monologues intérieurs de la littérature française" (éd. Pléiade, pp. 1243-1244). (Les deux monologues en question sont Mon plus secret conseil et Amants, heureux amants, l'innovation étant, dans ce dernier texte, beaucoup moins visible, puisqu'il est principalement écrit en je).

À la suite de cette curieuse reconnaissance d'une dette en quelque sorte rétrospective, il s'ensuivit une correspondance, dont on peut relever certains éléments saillants: si Dujardin n'avait pas persévéré dans la forme des Lauriers..., c'est qu'il n'en avait plus vu que les "défauts" (en outre, le livre n'avait pas reçu un très bon accueil $\left.{ }^{9}\right)$. La dernière lettre à V. Larbaud (19 avril 1931) comporte des indications hautement significatives : "Merci pour ce que vous m'écrivez du monologue intérieur à la troisième personne [...] En dehors du monologue intérieur proprement dit qui serait à la troisième personne, il y a quelque chose qui me semble également possible et dont je crois qu'il existe des essais. Voici : c'est celui où, à la vérité, l'auteur, et non le personnage, parle et par conséquent dit: "IL", mais d'une façon tellement objective qu'il ne fait en somme qu'exprimer la pensée profonde du personnage; en deux mots, l'analogue de ce qu'en littérature classique on appelle le "discours indirect". Dans ce cas, le "IL" est en réalité un "JE" dissimulé" (éd. Pléiade, p. 1245). Or ce qu'en 1931 Dujardin envisage comme une possibilité théorique définit on ne peut plus exactement la technique effectivement utilisée par Larbaud en 1922-1923 !

On pourra rêver indéfiniment sur l'énigmatique mention, par Dujardin, des "défauts" du monologue intérieur ... Un tel discours nous semble

\footnotetext{
${ }^{8}$ La dette de reconnaissance dont Saussure se croit redevable à Whitney ne s'explique pas autrement : par une espèce de coup de foudre.

${ }^{9}$ Ce qui ne l'empêchera pas d'être régulièrement réédité jusqu'aujourd'hui.
} 


\section{Francis Gandon}

symboliser la catégorie même du romanesque. Catégorie impure en ce qu'elle propose une sorte d'inceste syntaxique (l'appariement du je et de l'aoriste) ; mais surtout en ce qu'elle permet à la non-personne d'exprimer objectivement le subjectivisme le plus débridé et assujettit l'instance d'énonciation à un principe d'indétermination. La perversion de l'axe pronominal ne peut aboutir, du point de vue du linguiste et de Benveniste, qu'à la perte d'un paradigme essentiel. Donc à une désorientation majeure. C'est ainsi une raison analogue, dans son principe, à celle qui rejette les "sens opposés dans les mots primitifs", qui exclut le romanesque, et ses "pronoms indistincts", hors de la norme scientifique.

\section{Bibliographie}

BANFIELD, Ann, 1973. " Le style narratif et la grammaire des discours direct et indirect ". In La critique générative, Paris, Seghers-Laffont.

BENVENISTE, Émile, 1959. " Les relations de temps dans le verbe français ". Bulletin de la société de linguistique de Paris, LIV, fasc. I. In Problèmes de linguistique générale I, Paris, Gallimard, 1966.

1970." L'appareil formel de l'énonciation ". Langages, Paris, Didier-Larousse. In Problèmes de linguistique générale II, Paris, Gallimard, 1974.

DiALO, Amadou, 1983. Éléments systématiques du wolof contemporain. Mémoire (inédit), Centre de linguistique appliquée de Dakar.

DUJARDIN, Émile, 1887. Les lauriers sont coupés. Paris, Revue indépendante.

GREIMAS, Algirdas-Julien, 1972. " Pour une théorie du discours poétique". In Essais de sémiotique poétique. Paris, Larousse.

GREIMAS, A.J. - COURTÈs, Joseph, 1979. Sémiotique. Dictionnaire raisonné de la théorie du langage. Paris, Hachette.

HJELMSLEV, Louis, 1943. Prolégomènes à une théorie du langage. Paris, Éd. de minuit, 1968-1971.

LARBAUD, Valéry, 1984. Oeuvres complètes. Paris, Gallimard, Bibliothèque de la Pléïade.

MALKA, Jean-Guy, 1990. La langue Hausa (parler de Filingué) : Grammaire. Thèse (inédite) de doctorat d'État, Paris, Sorbonne.

MILNER, Jean-Claude, 1978. De la syntaxe à l'interprétation. Paris, Le Seuil.

MOESCHLER, Jacques, 1993. "Aspects pragmatiques de la référence temporelle : indétermination, ordre temporel et inférence", Langages ${ }^{\circ} 112$. Paris, Larousse.

PROST, André (R.P.), 1979. Le baatonum. Une langue voltaïque en voie de disparition. Mémoire (inédit) , Faculté des Lettres de Dakar, Département de linguistique.

RUWET, Nicolas, 1982. Grammaire des insultes et autres études. Paris, Le Seuil.

VuILLAUME, Marcel, 1990. Grammaire temporelle des récits. Paris, Minuit.

WeINRICH, Harald, 1963. Le Temps. Paris, Le Seuil, 1973. 